\title{
Genome-wide analysis of DNA methylation and gene expression changes in an ovalbumin-induced asthma mouse model
}

\author{
JOONG-SUN KIM ${ }^{1 *}$, IN-SIK SHIN ${ }^{2 *}$, NA-RAE SHIN ${ }^{2}$, JAE-YONG NAM ${ }^{3}$ and CHUL KIM ${ }^{4}$ \\ ${ }^{1}$ Herbal Medicine Resources Research Center, Korea Institute of Oriental Medicine, Naju-si, Jeollanam-do 58245; \\ ${ }^{2}$ College of Veterinary Medicine and BK21 Plus Project Team, Chonnam National University, Gwangju 61186; \\ ${ }^{3}$ Research and Development Center, Insilicogen Inc., Yongin-si, Gyeonggi-do 16954; ${ }^{4}$ Future Medicine Division, \\ Korea Institute of Oriental Medicine, Daejeon 34054, Republic of Korea
}

Received August 2, 2019; Accepted February 4, 2020

DOI: $10.3892 / \mathrm{mmr} .2020 .11245$

\begin{abstract}
The aim of the present study was to establish an integrated network of DNA methylation and RNA expression in an ovalbumin (OVA)-induced asthma model, and to investigate the epigenetically-regulated genes involved in asthma development. Genome-wide CpG-DNA methylation profiling was conducted through the use of a methylated DNA immunoprecipitation microarray and RNA sequencing was performed using three lung samples from mice with OVA-induced asthma. A total of 35,401 differentially methylated regions (DMRs) were identified between mice with OVA-induced asthma and control mice. Of these, 3,060 were located in promoter regions and 370 of the genes containing these DMRs demonstrated an inverse correlation between methylation and gene expression. Kyoto Encyclopedia of Genes and Genomes pathway analysis identified that 368 genes were upregulated or downregulated in OVA-induced asthma samples, including genes involved in 'chemokine signalling pathway', 'focal adhesion', 'leukocyte transendothelial migration' and 'vascular smooth muscle contraction signaling' pathways. Integrated network analysis identified four hub genes, consisting of three upregulated genes [forkhead box O1 (FOXO1), SP1 transcription factor (SPI) and amyloid $\beta$ precursor protein $(A P P)]$, and one downregulated gene [RUNX family transcription factor $1(R U N X 1)]$, all of which demonstrated an association between DNA methylation and gene expression. These genes were highly interconnected nodes in the Ingenuity Pathway Analysis module and were functionally significant. A total of four interconnected hub genes, FOXO1, RUNX1, SPI and APP, were identified from the integrated DNA methylation and gene expression networks involved in asthma development. These results suggested that
\end{abstract}

Correspondence to: Dr Chul Kim, Future Medicine Division, Korea Institute of Oriental Medicine, 1672 Yuseong-daero, Yuseong-gu, Daejeon 34054, Republic of Korea

E-mail: chulnice@kiom.re.kr

*Contributed equally

Key words: asthma, DNA methylation, gene expression, mice modulating these four genes could effectively control the development of asthma.

\section{Introduction}

Asthma is a chronic inflammatory airway disease that is considered an important public health concern. It usually develops in response to the inhalation of allergens, inhalants and air pollutants, and is characterized by pulmonary inflammation, airway hyper-responsiveness and mucus overproduction (1). Asthma and allergic diseases are considered to be complex genetic diseases caused by interactions between various genes and environmental factors, which are very important in the pathogenesis of asthma (2). These gene-by-environment interactions can be explained by the phenomenon of epigenetic regulation, which has provided mechanistic explanations linking molecular events and early-life environmental exposure with subsequent disease development (3). Epigenetic markers have emerged as potential participants in the mechanism of asthma, and have been demonstrated to modulate various immune cell processes involved in the development of disease (4-7). Furthermore, several previous epigenomic and transcriptomic studies have identified DNA methylation as a biomarker involved in the development of asthma $(8,9)$. Numerous asthma-promoting environmental factors can alter the epigenetic profiles of airway cells, suggesting that environmental exposure may cause asthma or contribute to phenotypic heterogeneity changes via DNA methylation (10-12).

Experimental asthma models have been established using a variety of stimuli, such as house dust mites, ovalbumin (OVA), fungi, cockroach extracts, ragweed and latex (13). These animal models have focused on the role of type 2 immune responses driven by allergic reactions in the development of asthma (14). An OVA-induced asthma model was developed to evaluate the efficacy of therapeutic agents prior to clinical trials and to investigate their mechanisms of action; it has been used to identify the signaling pathways involved in the development of asthma (15). Additionally, the OVA-induced asthma model has often been used to identify the epigenetic mechanisms of therapeutic agents and the pathogenesis of asthma (16-18); however, to the best of the authors' knowledge, no studies have explained the association between DNA methylation and gene expression in the development of OVA-induced asthma. 
The present study aimed to establish an integrated network of DNA methylation and RNA expression in an OVA-induced asthma model and to investigate the involvement of epigenetically-regulated genes related to the development of asthma.

\section{Materials and methods}

Animals. A total of 6 female BALB/c mice ( 8 weeks old and weight 20-25 g) were obtained from Daehan BioLink Co., Ltd., and were housed under standard laboratory conditions $\left(22 \pm 2^{\circ} \mathrm{C}, 60 \%\right.$ relative humidity, $12 / 12 \mathrm{~h}$ light/dark cycle, and food and water ad libitum) for at least 1 week before the experiment began. Ethical and scientific management procedures for all animals were approved by The Institutional Animal Care and Use Committee of the Korea Institute of Oriental Medicine (approval no. 17-073).

Induction of asthma using OVA. Mice were randomly divided into two groups: Vehicle-treated $(n=3)$ and OVA-treated $(n=3)$. Asthma was induced using OVA according to the previously described experimental procedures (19). To boost immune responses, mice were injected intraperitoneally with OVA (50 $\mu \mathrm{g})$ emulsified with $200 \mu \mathrm{l}$ aluminum sulfate (InvivoGen) twice, 7 days apart (on day 0 and 7). The mice were administered intranasally with $50 \mu \mathrm{l}$ OVA $(25 \mu \mathrm{g})$ from day 12 to 15 under anesthesia with $2 \%$ isoflurane (Piramal Critical Care, Ltd.).

Evidence of OVA-induced asthma. Pathophysiological evidence of OVA-induced asthma was indicated by airway inflammation, eosinophil and interleukin (IL)-4 production (Fig. S1). The right lungs of the mice were fixed with $10 \%$ neutral buffered formalin at $25^{\circ} \mathrm{C}$ for $24 \mathrm{~h}$, embedded in paraffin, sectioned into $4-\mu \mathrm{m}$ thick slices, and stained with hematoxylin for $5 \mathrm{~min}$ and eosin $3 \mathrm{~min}$ (Sigma-Aldrich; Merck $\mathrm{KGaA}$ ) at $25^{\circ} \mathrm{C}$ to evaluate inflammatory responses in lung tissues, as well as with periodic acid-Schiff (IMEB Inc.) to evaluate mucus production. A tracheostomy was performed in mice anesthetized with $85 \mathrm{mg} / \mathrm{kg}$ alfaxan (Alfaxalone ${ }^{\circledR}$; Jurox Pty Ltd.) to obtain bronchoalveolar lavage fluid (BALF). The lungs were lavaged twice with cold PBS (total volume $1.4 \mathrm{ml}$ ) and the obtained BALF was centrifuged at $380 \times \mathrm{g}$ for $5 \mathrm{~min}$ at $4^{\circ} \mathrm{C}$. The supernatant was used for an ELISA assay (cat. no. M400B; R\&D Systems, Inc.) in order to analyze level of IL-4, according to the manufacturer's protocol. The pellets of BALF were used to count inflammatory cells in the BALF. The eosinophil count was determined using a cell counting machine (Countess II $^{\mathrm{TM}}$ Automated Cell Counter, Thermo Fisher Scientific, Inc.).

Statistical analysis. Statistical analysis was performed using Student's t-test with Prism 8 software (GraphPad Software, Inc.). $\mathrm{P}<0.05$ was considered to indicate a statistically significant difference.

Microarray data. A schematic diagram for identifying target genes involved in asthma development is presented in Fig. 1. Gene expression profile data was used from a previous study by Baek et al (18), which were deposited in Gene Expression Omnibus (GSE114587; https:/www.ncbi.nlm.nih.gov/geo/ query/acc.cgi?acc=GSE114587). A total of six chips were available for further analysis, including samples from three vehicle- and three OVA-treated animals. Detailed information is available from the GEO database (18).

RNA-sequencing (RNA-seq) and data analysis. Total RNA was extracted from the lung tissues of saline-treated (normal) and OVA-induced mice. RNA isolation was performed using the PureLink $^{\mathrm{TM}}$ RNA Mini kit (cat. no. 12183018A; Thermo Fisher Scientific, Inc.). RNA-seq libraries were prepared using the TruSeq RNA Sample Prep kit (Illumina, Inc.) and sequenced with a NextSeq ${ }^{\circledR} 500$ System Whole-Genome Sequencing Solution (Illumina, Inc.) using the 76-bp paired-end reads. The raw FASTQ reads were trimmed to remove adapters and low-quality reads (per-base quality <20) using cutadapt (v.1.10, https://cutadapt.readthedocs.io/en/stable/) (20), then the high-quality sequence reads were aligned to the Mus musculus genome (mm10) using HISAT2 (v.2.1.0) and StringTie (v.1.3.4, https://ccb.jhu.edu/software/stringtie/) (21). Gene expression was quantified using the ballgown package in R (v.2.6.0) (22). Differentially expressed genes (DEGs) between the normal and OVA-induced mice groups were identified using the edgeR package (v.3.16.5) (23) based on negative binomial models of RNA-seq count data. Candidate DEGs were filtered using $\log _{2}(\mathrm{FC}) \geq 11 \mathrm{l}$ and $\mathrm{P}<0.05$ as thresholds, where FC indicates the fold change. DAVID was used to functionally annotate the genes (Tables SI and SII) $(24,25)$.

Methyl-sequencing and data analysis. DNA methylation profile data from the previous study by Baek et al (18) was used. DNA libraries were prepared according to the SureSelectXT Methyl-Seq Target Enrichment System protocol (Agilent Technologies, Inc.) and sequenced using an Illumina HiSeq 2500 platform (Illumina, Inc.) to generate 101-bp paired-end reads. After sequencing, the raw FASTQ reads were trimmed to remove adapters and low-quality reads (per-base quality <20) using cutadapt (v.1.10) and mapped onto the bisulfite converted Mus musculus genome (mm10) using Bismark v.0.19.0 (https://www.bioinformatics.babraham.ac.uk/ projects/bismark/). The methylKit R package (v.1.6.0) (26) was used to analyze DNA methylation. Differentially methylated regions (DMRs) are presented in Fig. 2. The base-pair methylation profile was summarized over a tiling window (window size: 1,000 bp and step-size: $500 \mathrm{bp}$ ). Statistically significant DMRs were selected based on their Q-value and the percentage of methylation difference (CpGs, mean methylation difference in groups $>10 \%$; $\mathrm{Q}<0.01$ ). The genomation $\mathrm{R}$ package (27) was used to annotate the genomic features of DMRs, which were classified into six types (intergenic, 5'UTR, 3'UTR, promoter, CDS and intron) based on University of California Santa Cruz Genome Browser (28).

Combined analysis of DNA methylation and RNA expression. DNA methylation and gene expression are known to be closely related. To identify the genes whose RNA expression levels were affected by DNA methylation (i.e. inverse correlation between RNA expression and DNA methylation) in asthmatic mice, the expression level was adjusted to between 0 and 1 (as methylation $\beta$ values range between 0 and 1). Spearman's rank correlation coefficient was used to evaluate the correlation between $\mathrm{CpG}$ methylation and gene expression. To understand 


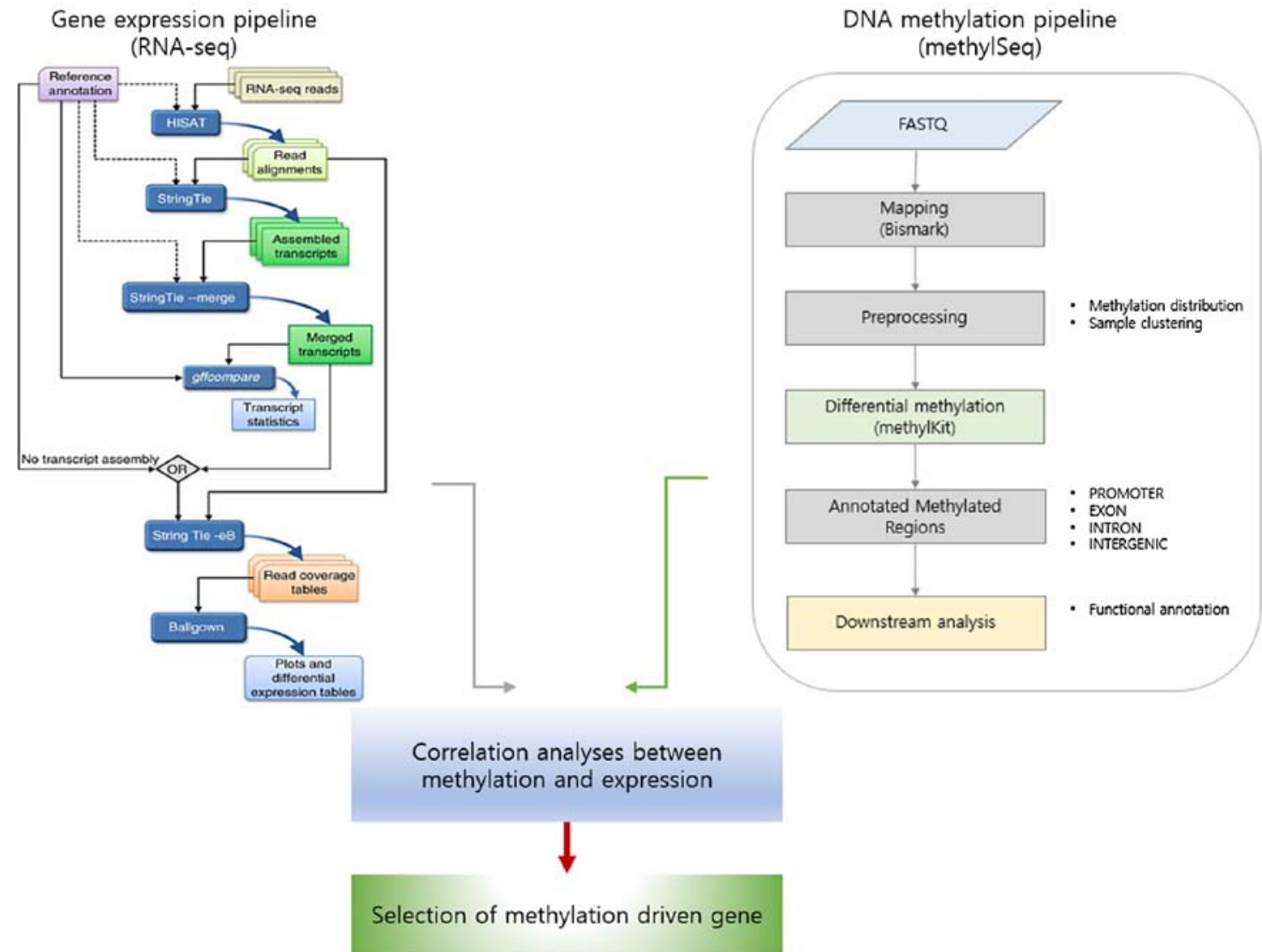

Figure 1. Schematic diagram for identifying target genes involved in asthma development. Gene Expression Omnibus (GSE114587; https://www.ncbi.nlm.nih. gov/geo/query/acc.cgi?acc=GSE114587). RNA-seq, RNA-sequencing.

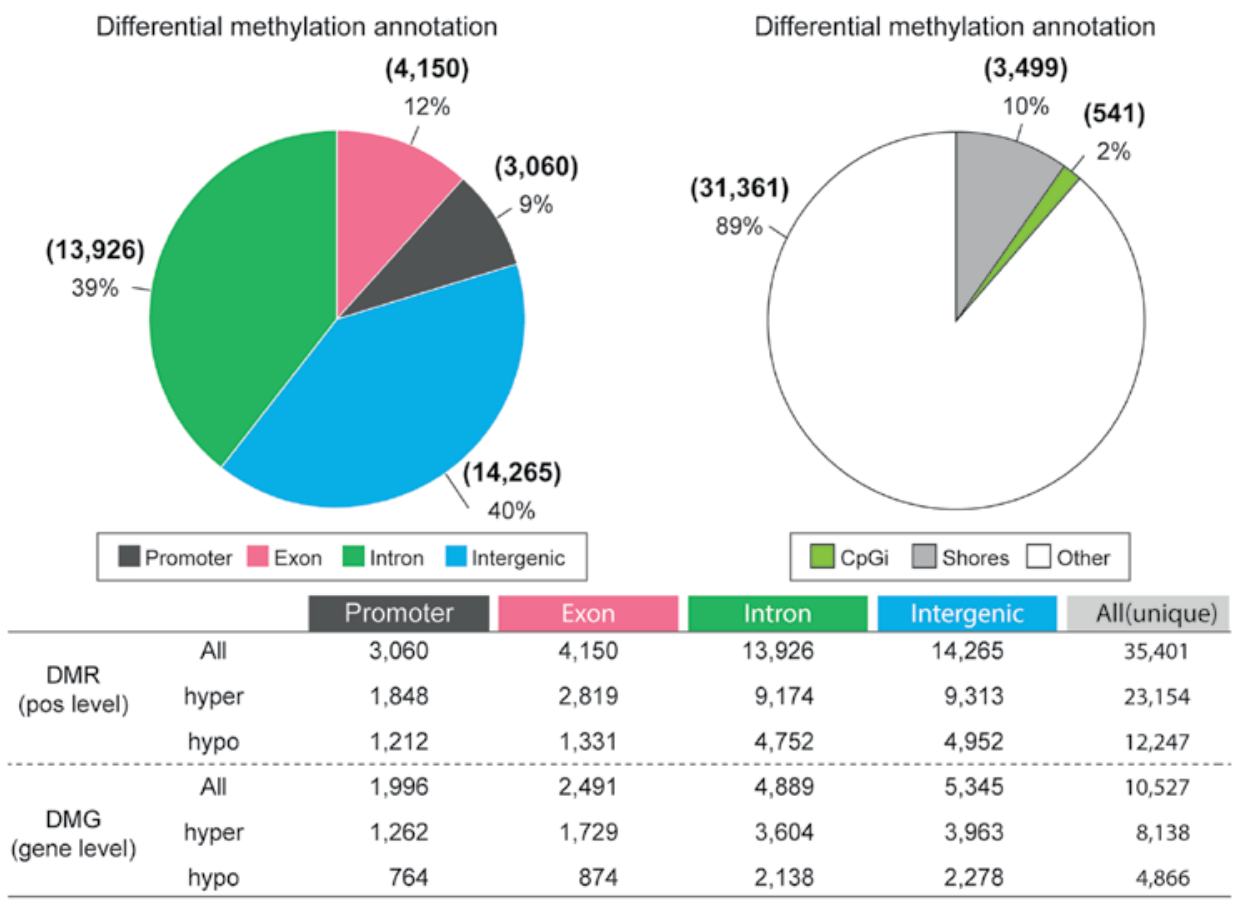

Figure 2. Genome-wide methylation profile of DMRs. DNA methylation profile data was used from the previous study by Baek et al (18). DNA libraries were prepared according to the SureSelectXT Methyl-Seq Target Enrichment System Protocol. CpGi, CpG islands; DMR, differentially methylated region; DMG, differentially methylated gene.

the biological mechanisms and pathways related to these genes in asthma, Gene Ontology (GO) enrichment analysis $(29,30)$ and Kyoto Encyclopedia of Genes and Genomes (KEGG) pathway (31) mapping were conducted using DAVID (24). 
Table I. Hyper-KEGG pathway functional annotation of DNA methylation.

\begin{tabular}{|c|c|c|c|}
\hline Term & Hit & Total & P-value \\
\hline Olfactory transduction & 13 & 389 & $3.80 \times 10^{-141}$ \\
\hline Cytokine-cytokine receptor interaction & 89 & 267 & $1.22 \times 10^{-43}$ \\
\hline Neuroactive ligand receptor interaction & 98 & 272 & $1.08 \times 10^{-41}$ \\
\hline Pathways in cancer & 170 & 328 & $4.18 \times 10^{-34}$ \\
\hline Systemic lupus erythematosus & 25 & 140 & $8.18 \times 10^{-34}$ \\
\hline Huntington's disease & 60 & 185 & $4.45 \times 10^{-31}$ \\
\hline MAPK signalling pathway & 129 & 267 & $2.51 \times 10^{-30}$ \\
\hline Oxidative phosphorylation & 30 & 135 & $2.68 \times 10^{-29}$ \\
\hline Chemokine signalling pathway & 70 & 190 & $1.13 \times 10^{-28}$ \\
\hline Alzheimer's disease & 55 & 169 & $1.66 \times 10^{-28}$ \\
\hline Natural killer cell mediated cytotoxicity & 36 & 137 & $5.31 \times 10^{-27}$ \\
\hline Parkinson's disease & 34 & 133 & $1.01 \times 10^{-26}$ \\
\hline Antigen processing and presentation & 10 & 89 & $5.96 \times 10^{-26}$ \\
\hline Spliceosome & 33 & 128 & $1.28 \times 10^{-25}$ \\
\hline Purine metabolism & 55 & 159 & $2.00 \times 10^{-25}$ \\
\hline Regulation of action cytoskeleton & 102 & 216 & $2.19 \times 10^{-25}$ \\
\hline Focal adhesion & 100 & 201 & $3.38 \times 10^{-22}$ \\
\hline Cell cycle & 41 & 128 & $4.23 \times 10^{-22}$ \\
\hline Lysosome & 36 & 121 & $5.19 \times 10^{-22}$ \\
\hline Endocytosis & 87 & 183 & $1.66 \times 10^{-21}$ \\
\hline
\end{tabular}

8,138 genes; Gene Ontology gene set (min. size=3; max. size=1,000); 1,235 significant KEGG pathways with $\mathrm{P}<0.01$. KEGG, Kyoto Encyclopaedia of Genes and Genomes.

Pathway analysis. Ingenuity Pathway Analysis (IPA; v.1.13, Qiagen,Inc.) software was used to predict putative sub-networks containing the four candidate genes, whose expression altered due to changes in DNA methylation in asthma. Briefly, the Molecule Activity Predictor tool in IPA was used to interrogate sub-networks using the methylation-expression candidate genes, then integrated the sub-network by overlaying the genes involved in asthma. To examine how each gene affected the sub-network, the fold change of gene expression and DNA methylation $\beta$ values were included.

Data access. The data from the present study were deposited in the GEO under accession number GSE114587 (https://www. ncbi.nlm.nih.gov/geo/query/acc.cgi?acc=GSE114587; secure token for reviewer: itytgkuoptclnej) (18).

\section{Results}

Methyl-sequencing exhibits DNA methylation alterations between vehicle-treated mice and mice with OVA-induced asthma. The DNA methylation of samples from the same animals were profiled in order to identify DMRs between the vehicle and the OVA-induced asthma samples (Fig. 2). After filtering the methylation profile, a total of 35,401 DMRs (base pair-level) were identified between the OVA-induced asthma and the saline-treated mice. Of these, 3,060 were located in promoter regions and 370 of the genes containing these DMRs demonstrated an inverse correlation between methylation and gene expression. Additionally, the number of hyper-methylated regions was twice as high as the number of hypo-methylated regions. Nevertheless, the proportion of functional regions, such as promoters and exons, was similar between the hyperand hypo-methylated regions (hyper-methylated: $20.1 \%$; hypo-methylated: $20.7 \%$ ). The regional distribution of DMRs was assessed based on their distance from the nearest $\mathrm{CpG}$ island (CpGi); shores were defined as the regions flanking the CpGi (0-2 kb range). The majority of DMRs identified in mice with OVA-induced asthma were located in other regions (89\% non-CpGi and non-shore); this was followed by shores (10\%) and CpGi (2\%). The basepair-level DMRs were combined with the differentially methylated genes to perform an integrated analysis of methylation and gene expression. The number of hyper-methylated genes was also higher than the number of hypo-methylated genes. These DMRs, which were verified as important genes, were used to infer the mechanisms underlying the development of asthma. DAVID was used to functionally annotate the genes (Tables I and II). KEGG pathway analysis identified that 1,235 genes were more highly expressed in the OVA-induced asthma samples, than in the vehicle samples, and were mainly involved in 'chemokine signalling pathway', 'cytokine-cytokine receptor interaction', 'MAPK signalling pathway', 'neuroactive ligand receptor interaction', 'olfactory transduction' and 'oxidative phosphorylation' pathways (Table I). Conversely, 170 genes were expressed at lower levels in the OVA-induced asthma samples than in the vehicle samples, and were enriched in the 'cytokine-cytokine receptor interaction', MAPK signalling pathway', 'neuroactive ligand receptor interaction' and 'olfactory transduction' pathways (Table II). 
Table II. Hypo-KEGG pathway functional annotation of DNA methylation.

\begin{tabular}{lrrr}
\hline Term & Hit & Total & P-value \\
\hline Olfactory transduction & 7 & 389 & $5.65 \times 10^{-102}$ \\
Neuroactive ligand receptor interaction & 55 & 272 & $5.59 \times 10^{-33}$ \\
Systemic lupus erythematosus & 14 & 140 & $1.07 \times 10^{-25}$ \\
Cytokine-cytokine receptor interaction & 72 & 267 & $5.55 \times 10^{-25}$ \\
Pathways in cancer & 113 & 328 & $9.82 \times 10^{-23}$ \\
Huntington's disease & 38 & 185 & $1.69 \times 10^{-22}$ \\
Alzheimer's disease & 31 & 169 & $2.02 \times 10^{-22}$ \\
Oxidative phosphorylation & 20 & 135 & $1.04 \times 10^{-20}$ \\
MAPK signalling pathway & 88 & 267 & 133 \\
Parkinson's disease & 22 & 128 & $5.00 \times 10^{-19}$ \\
Spliceosome & 22 & 72 & $4.98 \times 10^{-19}$ \\
Drug metabolism cytochrome P450 & 3 & 216 & $4.89 \times 10^{-18}$ \\
Regulation of action cytoskeleton & 69 & 89 & $2.35 \times 10^{-17}$ \\
Antigen processing and presentation & 9 & 138 & $7.53 \times 10^{-17}$ \\
Ubiquitin mediated proteolysis & 30 & 159 & $1.05 \times 10^{-16}$ \\
Purine metabolism & 41 & 178 & $2.40 \times 10^{-16}$ \\
Calcium signalling pathway & 52 & 128 & $6.54 \times 10^{-16}$ \\
Cell cycle & 27 & 183 & $1.37 \times 10^{-15}$ \\
Endocytosis & 57 & 55 & $1.63 \times 10^{-15}$ \\
Steroid hormone biosynthesis & 1 & $6.20 \times 10^{-15}$ \\
\hline
\end{tabular}

4,866 genes; Gene Ontology gene set ( $\min$. size $=3$; max. size $=1,000$ ); 170 significant KEGG pathways with $\mathrm{P}<0.01$. KEGG, Kyoto Encyclopaedia of Genes and Genomes.

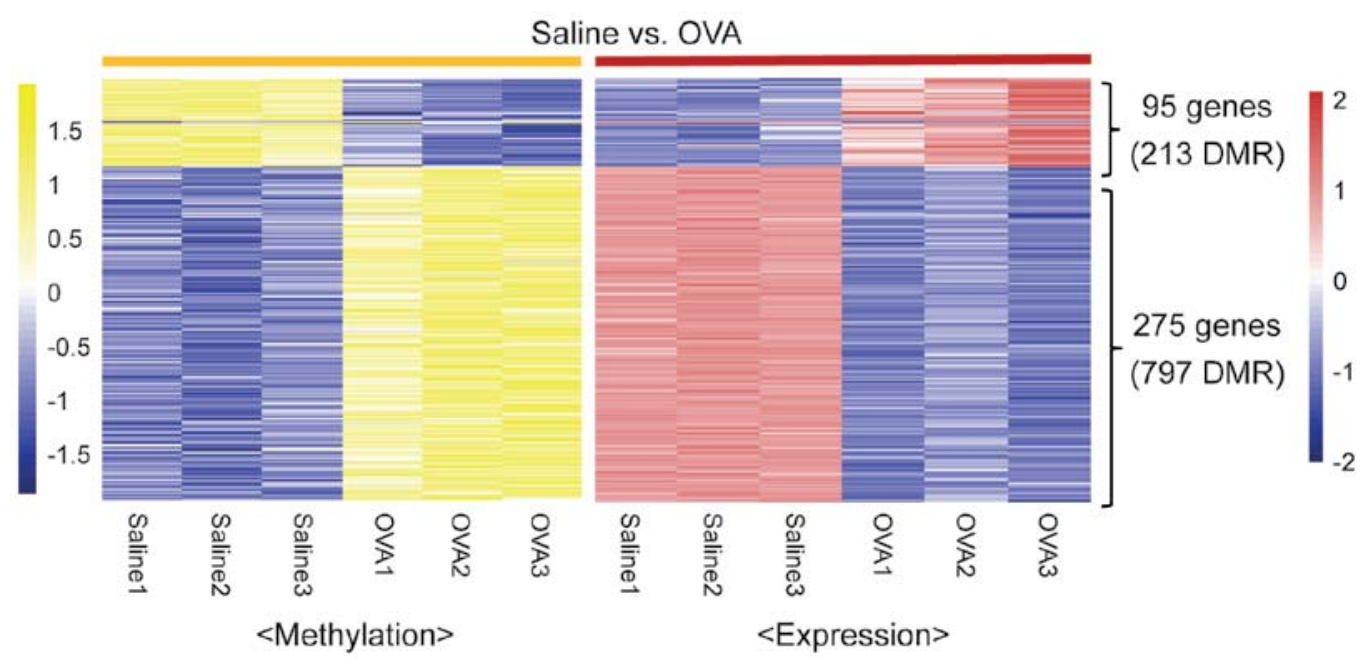

Figure 3. Correlation analysis between DNA methylation and gene expression. Gene Ontology enrichment analysis and Kyoto Encyclopedia of Genes and Genomes pathway mapping using DAVID. OVA, ovalbumin; DMR, differentially methylated region. Yellow indicates hyper-methylation and blue indicates hypo-methylation in the methylation heatmap. Red indicates upregulation and blue indicates downregulation in the expression heatmap.

Identification of epigenetically-regulated genes in the development of asthma. To verify the genes modulated by DNA methylation in the development of asthma, the RNA-seq data were analyzed for correlations between DNA methylation and gene expression (Fig. 3). A total of 95 genes were upregulated, whilst 275 genes were downregulated in the OVA-induced asthma samples. DAVID was used to functionally annotate the genes. KEGG pathway analysis identified that 368 genes were upregulated or downregulated in the OVA-induced asthma samples, and were mainly involved in 'acute myeloid leukemia', 'chemokine signalling pathway', 'focal adhesion', 'leukocyte transendothelial migration', 'vascular smooth muscle contraction' and 'neurotrophin signalling pathway' (Table III).

Identification of four genes associated with asthma development using integrated network analysis. An integrated 
Table III. Kyoto Encyclopaedia of Genes and Genomes pathway functional annotation of DNA methylation and gene expression.

\begin{tabular}{lrrr}
\hline Term & Count & $\%$ & P-value \\
\hline mmu05221: Acute myeloid leukemia & 7 & 2.07715134 & 0.00156622 \\
mmu04062: Chemokine signalling pathway & 12 & 3.56083086 & 0.00232787 \\
mmu04510: Focal adhesion & 12 & 3.56083086 & 0.00446246 \\
mmu04670: Leukocyte transendothelial migration & 9 & 2.67062315 & 0.00486667 \\
mmu04270: Vascular smooth muscle contraction & 9 & 2.67062315 & 0.00511869 \\
mmu04070: Phosphatidylinositol signalling system & 7 & 2.07715134 & 0.00627478 \\
mmu04916: Melanogenesis & 8 & 2.37388724 & 0.00669627 \\
mmu04722: Neurotrophin signalling pathway & 9 & 2.67062315 & 0.00822449 \\
mmu05200: Pathways in cancer & 15 & 4.45103858 & 0.01234863 \\
mmu04666: Fc $\gamma$ R-mediated phagocytosis & 7 & 2.07715134 & 0.02170314 \\
mmu05212: Pancreatic cancer & 6 & 1.7841543 & 0.02172617 \\
mmu05213: Endometrial cancer & 5 & 1.48367953 & 0.02805151 \\
mmu00562: Inositol phosphate metabolism & 5 & 1.48367953 & 0.03168093 \\
mmu04512: ECM-receptor interaction & 6 & 1.78041543 & 0.03721468
\end{tabular}

Program: DAVID Functional Annotation tool; Input: 368 genes (correlation $<0$ and $\mathrm{P}<0.05$ ).
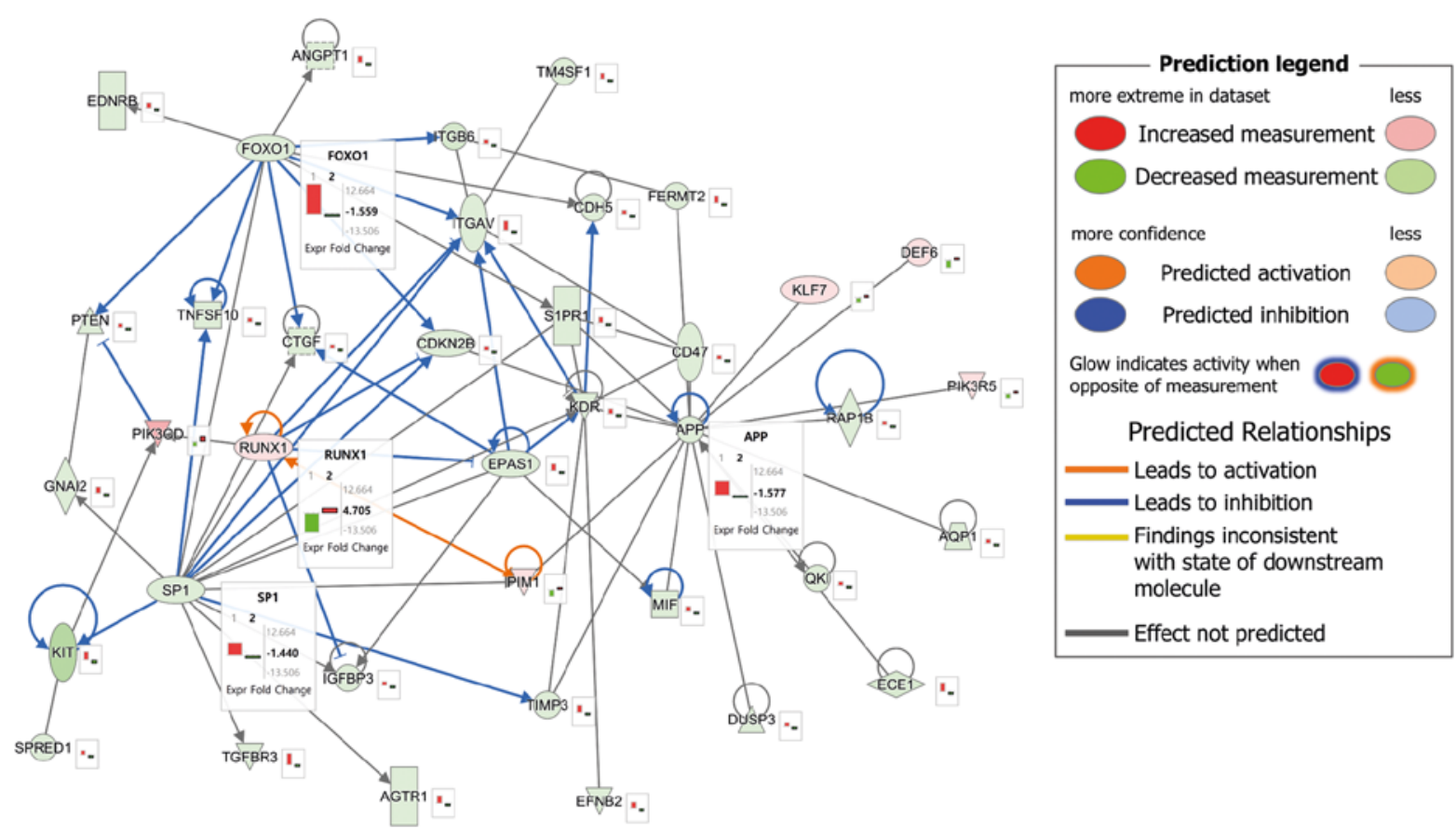

Figure 4. Gene networks involved in asthma development. Ingenuity Pathway Analysis software was used to predict putative sub-networks containing the four candidate genes. FOXO1, forkhead box O1; APP, amyloid $\beta$ precursor protein; RUNX1, RUNX family transcription factor 1; SP1, SP1 transcription factor.

network analysis of DNA methylation and gene expression was conducted to understand the interactions between the genes related to asthma development. The predicted pathway is presented in Fig. 4. There were four hub genes, consisting of three upregulated genes [forkhead box O1 (FOXO1), SP1 transcription factor $(S P 1)$ and amyloid $\beta$ precursor protein $(A P P)]$ and one downregulated gene [RUNX family transcription factor $1(R U N X 1)]$, which demonstrated the association between DNA methylation and gene expression. These genes were closely interconnected nodes in the IPA module, and were functionally significant. Therefore, FOXO1, SP1, APP and $R U N X 1$ were identified as key integrated network regulators in the development of asthma.

\section{Discussion}

The purpose of the present study was to conduct an in-depth analysis of DNA methylation and gene expression in the lung tissues of an OVA-induced asthma mouse model. The important features of DNA methylation in asthma development and how they differ from those of gene expression were investigated. The present results identified important genes 
underlying asthma development, and provided an incorporated network of DNA methylation and gene expression in mice with OVA-induced asthma.

Histopathology was conducted, and the eosinophil count and IL-4 level were determined, as indicators for establishment of asthma in animals. In the development of asthma, IL-4 leads to eosinophil-rich airway inflammation, elevated immunoglobulin E production and mucus hypersecretion by goblet cells (15). In addition, eosinophilia is considered as an important feature in allergic asthma. Activated eosinophils, as a result of various allergens, can lead to changes in levels of reactive oxygen species, cationic granule proteins, growth factors and cytokines (32). Therefore, resulting in the aggravation of allergic responses, such as airway inflammation and mucus hypersecretion (13). In the present study, the experimental model demonstrated that the eosinophil count and IL-4 were increased with inflammatory cell infiltration into lung tissue in comparison with the normal controls. This suggested that allergic asthma was successfully established in the animal model.

The present study primarily profiled the genome-wide gene and methylation expression patterns of lung tissue from OVA-induced asthmatic animals described by Baek et al (18) in order to examine the effect of methylation on asthma development. GO analysis demonstrated that the candidate genes were associated with cytokines/chemokines, MAPKs and oxidative stress signaling pathways. Asthma is characterized by airway inflammation, hyper-responsiveness and mucus overproduction (32); airway inflammation is induced by various factors, including cytokines, chemokines, reactive oxygen species and MAPK signaling pathways, all of which accelerate the development of asthma (33). Cytokines and chemokines induce asthmatic pathophysiological alterations, such as eosinophilia, smooth muscle contraction and mucus hyperproduction (34). MAPK signaling also plays a crucial role in the development of asthma, and MAPK phosphorylation induces airway inflammation and mucus production by activating epidermal growth factor signaling (35).

GO analysis identified a strong enrichment of terms associated with acute myeloid leukemia, chemokines, focal adhesion and vascular smooth muscle contraction signaling pathways. Furthermore, FOXO1, RUNX1, SPI and APP were identified as closely connected hub genes in the integrated networks of DNA methylation and gene expression affecting the development of asthma. FOXO1 promotes inflammation by increasing the expression of several pro-inflammatory genes, including $I L-1 B, I L-6$ and $I L-13$, and exacerbates type 2 immune allergic airway inflammation in response to allergen challenge (36). In contrast, RUNXI mediates the normal maturation of immune system functions in various organisms; for example, RUNXI promotes $\mathrm{T}$ helper type 1 cell development by activating $I L-4$ silencers, reducing $I L-4$ expression, and elevating interferon- $\gamma$ expression (37); therefore, $R U N X 1$ dysfunction activates $\mathrm{T}$ helper type 2 cell immune responses and induces asthmatic phenotypes (37). SPI is the transcription factor of WNT-5A expression in airway smooth muscle cells, and its activity and expression are regulated by the phosphorylation of MAPKs, such as p38 and JNK (38).

Recently, it was identified that $A P P$ was associated with childhood-onset asthma in a network-assisted analysis of genome-wide association studies of asthma (39). Of the four genes, previous studies have demonstrated that $\mathrm{FOXO1}$ and $R U N X 1$ are correlated with the development of asthma $(37,38)$. However, to the best of the authors' knowledge, $S P 1$ and APP have not been studied extensively and therefore thee are insufficient data to correlate these genes with the development of asthma. In particular, in the case of $A P P$, only clinical values were provided and to the best of the authors' knowledge, no studies have been performed on the association with the development of asthma. The results of the present study may differ from those of previous studies. However, these differences are considered to be due to various experimental conditions, such as transgenic, knockout mice and stimulants. For example, in the case of aquaporin-3, the elevation of its expression induced the development of asthma in a previous study (40). However, in another previous study, aquaporin-3 expression was not altered compared with normal controls in the OVA-induced asthma model and was only upregulated in an IL-13-induced asthma model (41). As such, small differences may be identified depending on the respective experimental conditions. Therefore, further studies are needed to elucidate the association between these two genes and the development of asthma.

In summary, the present study focused on RNA-DNA methylation, which is one of the factors that can affect RNA expression in the development of asthma. In the present study, the genes that were highly associated with both RNA expression and DNA methylation were analyzed in the OVA-induced asthma model. FOXO1, RUNX1, SP1 and APP were identified as closely connected hub genes in an integrated network of DNA methylation and gene expression affecting the development of asthma. The results of the present study suggested that the modulation of these four genes could contribute to the development of asthma.

\section{Acknowledgements}

Not applicable.

\section{Funding}

This study was supported by The Application Development of Standardized Herbal Resources (grant no. KSN1911420) from The Korea Institute of Oriental Medicine.

\section{Availability of data and materials}

The datasets used and/or analyzed during the current study are available from the corresponding author on reasonable request.

\section{Authors' contributions}

JSK and ISS drafted the manuscript. ISS, JSK and CK conceived and designed the study. NRS, JYN, JSK and ISS analyzed the data. JSK and CK reviewed and edited the manuscript. All authors read and approved the final manuscript.

\section{Ethics approval and consent to participate}

All experimental procedures were approved by The Institutional Animal Care and Use Committee of Korea Institute of Oriental 
Medicine (approval no. 17-073) and the animals were cared for in accordance with The National Institutes of Health Guide for the Care and Use of Laboratory Animals.

\section{Patient consent for publication}

Not applicable.

\section{Competing interests}

The authors declare that they have no competing interests.

\section{References}

1. Deliu M, Belgrave D, Sperrin M, Buchan I and Custovic A: Asthma phenotypes in childhood. Expert Rev Clin Immunol 13: 705-713, 2017.

2. Kanagaratham C and Radzioch D: Allergic asthma: A summary from genetic basis, mouse study, to diagnosis and treatment. Curr Pharm Des 22: 6261-6272, 2016.

3. Yang IV, Lozupone CA and Schwartz DA: The environment, epigenome, and asthma. J Allergy Clin Immunol 140: 14-23, 2017.

4. Karmaus W, Ziyab AH, Everson T and Holloway JW: Epigenetic mechanisms and models in the origins of asthma. Curr Opin Allergy Clin Immunol 13: 63-69, 2013.

5. Kuo CH, Hsieh CC, Lee MS, Chang KT, Kuo HF and Hung CH: Epigenetic regulation in allergic diseases and related studies. Asia Pac Allergy 4: 14-18, 2014.

6. Salam MT: Asthma epigenetics. Adv Exp Med Biol 795: 183-199, 2014.

7. Harb H, Alashkar Alhamwe B, Garn H, Renz H and Potaczek DP. Recent developments in epigenetics of pediatric asthma. Curr Opin Pediatr 28: 754-763, 2016.

8. Chan MA, Ciaccio CE, Gigliotti NM, Rezaiekhaligh M, Siedlik JA, Kennedy K and Barnes CS: DNA methylation levels associated with race and childhood asthma severity. J Asthma 54: 825-832, 2017.

9. Lund RJ, Osmala M, Malonzo M, Lukkarinen M, Leino A, Salmi J, Vuorikoski S, Turunen R, Vuorinen T, Akdis C, et al: Atopic asthma after rhinovirus-induced wheezing is associated with DNA methylation change in the SMAD3 gene promoter. Allergy 73: 1735-1740, 2018.

10. Zahiruddin AS, Grant JA and Sur S: Role of epigenetics and DNA-damage in asthma. Curr Opin Allergy Clin Immunol 18: 32-37, 2018

11. Potaczek DP, Harb H, Michel S, Alhamwe BA, Renz H and Tost J: Epigenetics and allergy: From basic mechanisms to clinical application. Epigenomics 9: 539-571, 2017.

12. Liang L, Willis-Owen SAG, Laprise C, Wong KCC, Davies GA, Hudson TJ, Binia A, Hopkin JM, Yang IV, Grundberg E, et al: An epigenome-wide association study of total serum immunoglobulin E concentration. Nature 520: 670-674, 2015.

13. Haspeslagh E, Debeuf N, Hammad H and Lambrecht BN: Murine models of allergic asthma. Methods Mol Biol 1559: 121-136, 2017.

14. Sagar S, Akbarshahi $\mathrm{H}$ and Uller L: Translation value of animal models of asthma: Challenges and promises. Eur J Pharmacol 759: 272-277, 2015.

15. Kianmeher M, Ghorani V and Boskabady MH: Animal model of asthma, various methods and measured parameters: A methodological review. Iran J Allergy Asthma Immunol 15: 445-465, 2016.

16. Wu CJ, Yang CY, Chen YH, Chen CM, Chen LC and Kuo ML: The DNA methylation inhibitor 5-azacytidine increases regulatory $T$ cells and alleviates airway inflammation in ovalbumin-sensitized mice. Int Arch Allergy Immunol 160: 356-364, 2013.

17. Xu XF, Hu QY, Liang LF, We L, Gu WZ, Tang LL, Fu LC and Du LZ: Epigenetics of hyper-responsiveness to allergen challenge following intrauterine growth retardation rat. Respir Res 15: 137, 2014.

18. Baek SJ, Chun JM, Kang TW, Seo YS, Kim SB, Seong B, Jang Y, Shin GH and Kim C: Identification of epigenetic mechanisms involved in the anti-asthmatic effects of Descurainia sophia seed extract based on a multi-omics approach. Molecules 23: 2879, 2018

19. Chun JM, Lee AR, Kim HS, Lee AY, Gu GJ, Moon BC and Kwon BI: Peucedanum japonicum extract attenuates allergic airway inflammation by inhibiting Th2 cell activation and production of pro-inflammatory mediators. J Ethnopharamcol 211: 78-88, 2018.
20. Martin M: Cutadapt removes adapter sequences from high-throughput sequencing reads. EMBnet J: 17, 2011.

21. Kim D, Langmead B and Salzberg SL: HISAT: A fast spliced aligner with low memory requirements. Nat Methods 12: 357-360, 2015.

22. Fu J, Frazee AC, Collado-Torres L, Jaffe AE and Leek JT: ballgown: Flexible, isoform-level differential expression analysis. R package version 2.6.0. 2016.

23. Robinson MD, McCarthy DJ and Smyth GK: edgeR: A bioconductor package for differential expression analysis of digital gene expression data. Bioinformatics 26: 139-140, 2010.

24. Huang da W, Sherman BT and Lempicki RA: Systematic and integrative analysis of large gene lists using DAVID bioinformatics resources. Nature Protoc 4: 44-57, 2009.

25. Huang Da W, Sherman BT and Lempicki RA: Bioinformatics enrichment tools: Paths toward the comprehensive functional analysis of large gene lists. Nucleic Acids Res 37: 1-13, 2009.

26. Akalin A, Kormaksson M,Li S, Garrett-Bakelman FE, Figueroa ME, Melnick A and Mason CE: methylKit: A comprehensive R package for the analysis of genome-wide DNA methylation profiles. Genome Biol 13: R87, 2012.

27. Akalin A, Franke V, Vlahoviček K, Mason C and Schübeler D: Genomation: A toolkit to summarize, annotate and visualize genomic intervals. Bioinformatics 31: 1127-1129, 2014.

28. Kent WJ, Sugnet CW, Furey TS, Roskin KM, Pringle TH, Zahler AM and Haussler D: The human genome browser at UCSC. Genome Res 12: 996-1006, 2002

29. Ashburner M, Ball CA, Blake JA, Botstein D, Butler H, Cherry JM, Davis AP, Dolinski K, Dwight SS, Eppig JT, et al: Gene ontology: Tool for the unification of biology. The Gene Ontology Consortium. Nat Genet 25: 25-29, 2000

30. The Gene Ontology Consortium: The Gene Ontology Resource: 20 years and still GOing strong. Nucleic Acids Res 47: D330-D338, 2019.

31. Shin NR, Kwon HJ, Ko JW, Lee IC, Kim JC, Kim SH and Shin IS: S-allyl cysteine reduces eosinophilic airway inflammation and mucus overproduction on ovalbumin-induced allergic asthma model. Int Immunopharmacol 68: 124-130, 2019.

32. Aqhasafari P, George U and Pidaparti R: A review of inflammatory mechanism in airway disease. Inflamm Res 68: 59-74, 2019.

33. Lambrecht BN, Hammad H and Fahy JV: The cytokines of asthma. Immunity 50: 975-991, 2019

34. Khorasanizadeh M, Eskian M, Gelfand EW and Rezaei N: Mitogen-activated protein kinases as therapeutic targets for asthma. Pharmacol Ther 174: 112-126, 2017.

35. Shin NR, Ryu HW, Ko JW, Park SH, Yuk HJ, Kim HJ, Kim JC, Jeong S and Shin IS: Artemisia argyi attenuates airway inflammation in ovalbumin-induced asthmatic animals. J Ethnopharmacol 209: 108-115, 2017.

36. Chung S, Lee TJ, Reader BF, Kim JY, Lee YG, Park GY, Karpurapu M, Ballinger MN, Qian F, Rusu L, et al: FoxO1 regulates allergic asthmatic inflammation through regulating polarization of the macrophage inflammatory phenotype. Oncotarget 7 : 17532-17546, 2016.

37. Haley KJ, Lasky-Su J, Manoli SE, Smith LA, Shahsafaei A, Weiss ST and Tantisira K: RUNX transcription factors: Association with pediatric asthma and modulated by maternal smoking. Am J Physiol Lung Cell Mol Physiol 301: L693-L701, 2011.

38. Kumawat K, Menzen MH, Slegtenhorst RM, Halayko AJ, Schmidt M and Gosens R: TGF- $\beta$-induced WNT-5A expression in airway smooth muscle cells via Sp1 and $\beta$-catenin. PLoS One 9: e94801, 2014.

39. Liu Y, Brossard M, Sarnowski C, Vaysse A, Moffatt M, Margaritte-Jeannin P, Llinares-Lopez F, Dizier MH, Lathrop M, Cookson W, et al: Network-assisted analysis of GWAS data identifies a functionally-relevant gene module for childhood-onset asthma. Sci Rep 7: 938, 2017.

40. Ikezoe K, Oga T, Honda T, Hara-Chikuma M, Ma X, Tsuruyama T, Uno K, Fuchikami J, Tanizawa K. Handa T, et al: Aquaporin-3 potentiates allergic airway inflammation in ovalbumin-induced murine asthma. Sci Rep 6: 25781, 2016.

41. Kran CM, Deng B, Mutyam V, McDonald CA, Pazdziorko S, Masson L, Goldman S, Kasaian M, Chaudhary D, Williams C and Ho MW: Altered regulation of aquaporin gene expression in allergen and IL-13-induced mouse models of asthma. Cytokines 46: $111-118,2009$ International (CC BY-NC-ND 4.0) License. 